\title{
Processus téléologique et variabilité : Utilisation de la sensibilité au contexte
}

\section{Rébecca Deneckère, Elena Kornyshova}

Centre de Recherche en Informatique

Université Paris 1 Panthéon-Sorbonne, 90 rue de Tolbiac, 75013 Paris, France

\{rebecca.deneckere,elena.kornyshova\}@univ-paris1.fr

RÉSUMÉ. La variabilité s'est avérée être un concept central dans différents domaines d'ingénierie comme la fabrication, le développement de logiciels, etc. afin de développer des solutions pouvant être facilement adaptables à différents contextes organisationnels et/ou à différents types de clients pour un meilleur coût. Les processus téléologiques ont, par nature, un haut niveau de variabilité. Notre position est que l'intégration de la "sensibilité au contexte " dans ce type de processus permettra d'augmenter cette variabilité inhérente aux processus téléologiques avec l'ajout de critères spécifiques. Nous proposons une typologie d'indicateurs ainsi qu'un processus d'utilisation de ces critères et nous illustrons notre propos avec le méta-modèle de processus intentionnel MAP.

ABSTRACT. Variability has proved to be a central concept in different engineering domains, manufacturing, software development etc. in order to develop solutions that can be easily adapted to different organizational settings and different sets of customer at a low price. Teleological processes have, by nature, a high level of variability. Our position is that the integration of "context-awareness" in that kind of processes increases their intrinsic variability with specific criteria. We propose an indicator typology with an usage process and illustrate it with the MAP intentional process model.

MOTS-CLES Orienté-intention, Modèle de Processus, Variabilité, Sensibilité au contexte

KEYWORDS Intention-oriented, Process Model, Variability, Context-awareness 


\section{Introduction}

La variabilité s'est avérée être un concept central dans différents domaines d'ingénierie comme la fabrication, le développement de logiciels, etc. afin de développer des solutions pouvant être facilement adaptables à différents contextes organisationnels et/ou à différents types de clients pour un meilleur coût.

Les processus téléologiques sont des processus prenant en compte les intentions couplées aux résultats que l'utilisateur souhaite obtenir. D'un point de vue téléologique, tous les processus d'ingénierie de systèmes d'information (SI) contiennent des étapes de deux sortes: la prise de décision et l'exécution de la décision. Ces deux étapes alternent l'une avec l'autre. D'une part, la réalité téléologique de l'existence de processus exige des décisions. D'autre part, les décisions résultent de la nécessité de satisfaire des contraintes d'ordre pratique comme la qualité, les coûts ou les performances (Ruhe, 2003). Bien que ces aspects soient importants, les arguments pour prendre les décisions finales sont assez pauvres. Les choix sont effectués de manière intuitive et hasardeuse (Ruhe, 2003) (Ngo-The et al., 2005). La nature téléologique des processus se retrouve donc essentiellement dans les processus dits intentionnels. Ces processus sont flexibles par nature puisqu'ils prennent en compte les différents points de variation donnant lieu à un choix entre plusieurs intentions (Rolland, 2007). On peut donc dire qu'ils ont une variabilité intrinsèque à leur définition. Cette variabilité peut être augmentée avec la prise en compte de la variabilité due à la sensibilité au contexte.

Notre position est que l'intégration de la sensibilité au contexte dans ce type de processus permettra d'augmenter la variabilité inhérente aux processus téléologiques avec l'ajout de critères spécifiques. A cette fin, nous proposons une typologie d'indicateurs dont les valeurs permettent une prise de décision plus formelle. Nous illustrons notre proposition avec l'introduction de cette typologie dans le métamodèle de processus téléologique MAP.

Cet article est organisé comme suit. La section 2 détaille les notions de processus téléologiques et de variabilité. La section 3 approfondit le concept de sensibilité au contexte. Notre proposition est décrite dans la section 4 et illustrée dans la section 5 . La section 6 ouvre une discussion sur la notion de ligne de processus et nous concluons dans la section 7 .

\section{Les Processus Téléologiques et la Variabilité}

L'ingénierie des processus est considérée comme essentielle (Rolland, 1998) à la fois par la communauté de l'ingénierie des logiciels et par la communauté de l'ingénierie des systèmes. Par exemple, (Dowson et al., 1993) (Amenise et al., 1993) (Jarke et al., 1994) démontrent que l'amélioration d'un processus de 
développement conduira à une meilleure productivité et à une meilleure qualité des systèmes.

Les processus de développement traditionnels ont été conçus comme des suites d'étapes, chaque étape dominée par une activité particulière organisée en différentes séquences (par exemple, « l'analyse », « la conception », « le développement », « les tests » (Royce, 1970) (Boehm, 1988). Plus tard, ces séquences d'activités ont été remplacées par des valeurs et des pratiques (Beck, 2005) (Abrahamsson, 2002). (Ralph, 2008) met en évidence le fait que, dans la pratique, le développeur du logiciel est confronté à une myriade de choix méthodologiques, puisqu'il peut choisir d'exécuter une activité entièrement ou partiellement, combiner des aspects de deux ou plusieurs activités différentes, ou en ignorer une autre. Les décisions prises au cours de l'exécution conduisent l'ingénieur à adopter un processus qui est unique et complètement adapté à la situation en cours.

Toutefois, les points de décision ne sont souvent abordés que de manière partielle. La plupart des processus connus n'ont pas les moyens de prendre en compte la prise de décisions inhérente à tout processus de développement de systèmes d'information pour le développement. Selon Dowson (Dowson, 1988), les modèles de processus peuvent être classés en trois groupes de modèles appelés orientés-activités, orientés-produit et orientés-décision. (Rolland, 1998) raffine cette classification avec un quatrième groupe appelé orientés-contexte. Les processus orientés-décision et orientés-contexte prennent en compte la prise de décision en intégrant les concepts d'objectifs et d'arguments. Les modèles orientés-décision sont non seulement capables d'expliquer comment le processus est effectué, mais aussi pourquoi il est effectué (Rolland, 1998) (Ralph, 2008). Les modèles de processus orientés-contexte couplent la notion de contexte de décision à la décision elle-même (Grosz et al., 1997) (Rolland et al., 2000). Cela rend la notion de contexte (le couplage d'une situation et d'une décision) un concept central des processus de modélisation. Ces deux types de modèles de processus peuvent être définis comme des processus téléologiques.

Un processus téléologique est un processus qui prend en compte le comportement téléologique d'une exécution d'un processus (comportement attaché à la notion de but). Il décrit les intentions (buts, objectifs) associées à un résultat que le concepteur veut obtenir. (Taylor, 1964) propose une classification de comportement comme étant soit axé sur les buts (comportement téléologique) soit axé sur la réponse aux stimuli. (Malcom, 1967) définit un comportement téléologique comme suit: lorsqu'un comportement spécifique est nécessaire pour satisfaire un objectif, alors ce comportement se produit. En fait, il est nécessaire d'étudier la possibilité que les règles changent au cours du processus de développement. (Cayla, 2008) souligne le lien entre cette possibilité de changement et les principes téléologiques de Veblen (Veblen, 1898) qui intègrent les intentions de l'acteur dans les processus. Ce travail définit un processus téléologique comme « un équilibre dynamique dont les règles sont déterminées de façon endogène, comme une relation entre le comportement du système et de ses intentions ». Un tel 
processus téléologique peut exprimer les changements dans une trajectoire de processus. Par exemple, même si l'ingénieur a décidé d'un chemin général pour l'exécution de son processus, il a l'occasion de modifier ce chemin (au cours de l'exécution), en prenant en compte les informations et les connaissances qu'il a recueillies de la situation en cours.

(Van de Ven, 1995) définit les processus téléologiques comme décrivant le comportement des acteurs faisant des actions pour atteindre un but ou un objectif (un état final envisagé). (Ralph, 2008) souligne que cette théorie est compatible avec le développement de logiciels de la façon suivante : les agents téléologiques sont l'équipe de développement logiciel et le chef de projet; l'état final représente le produit logiciel, l'équipe de développement effectue des actions (codage, tests ...) qui correspondent aux étapes téléologiques et le chef de projet surveille les performances. La notion de but est également essentielle pour toute organisation. Le SI est crée car il y a plusieurs objectifs à atteindre et ses fonctions et propriétés sont définies par les objectifs de l'organisation que le système vise à remplir (Loucopoulos et al., 1995). Par exemple, les objectifs des processus sont envisagés dans des approches comme KAOS et $I^{*}$. Ces approches définissent la notion d'option alternative existant dans les processus d'ingénierie des exigences. Les intentions alternatives sont évaluées pour améliorer ces processus (Van Lamsweerde, 2009). Plus spécifiquement, dans I*, il existe un langage des exigences orienté objectif appelé GRL (Goal-oriented Requirement Language). Le GRL utilise les exigences non-fonctionnelles (NFR - Non-Functional Requirements). Ce langage permet d'identifier les objectifs et les solutions pour atteindre ces objectifs. Les solutions sont représentées comme des alternatives qui sont mesurées par rapport à leur contribution aux objectifs (Amyot et al., 2002).

Ces processus téléologiques intègrent, par le biais des décisions à prendre pour réaliser les intentions, une certaine variabilité. Au fil du temps, la Variabilité en génie logiciel est devenue de plus en plus importante. Dans les premiers temps, le SI permettait de réaliser les objectifs d'une seule organisation et d'un ensemble assez simple de clients, alors que, de nos jours, le SI doit être conçu dans une perspective plus large, pour répondre aux objectifs de plusieurs organisations, être adaptable à des situations et des clients différents (Rolland, 2007). La variabilité est la capacité à être sujet à la variation. La notion de variabilité du logiciel est donc définie comme la capacité d'un système logiciel à être modifié, adapté ou configuré dans un certain contexte (Van Gurp, 2000). Alors que la communauté du logiciel considère la variabilité comme un problème de conception et se concentre sur les questions d'implémentation (Svanberg, 2001) (Bosh et al., 2001) (Bachmann et al., 2001), nous estimons, comme (Halmans, 2003), que l'expression de la variabilité au niveau des buts est essentielle pour satisfaire la nature multi-objectifs de ces nouveaux SI, intégrant la variabilité et capables de s'auto-adapter à la situation en cours.

(Liaskos et al., 2007) établit que la modélisation de la variabilité est utile tant pour l'acquisition de variabilité - pour découvrir des points de variation - que pour l'analyse de la variabilité - pour évaluer l'applicabilité de chaque variant identifié 
5 Revue Ingénierie des Systèmes d'information.

dans un contexte et une situation donnés. Le grand nombre de caractéristiques observées dans les systèmes informatiques modernes, ainsi que la difficulté à comprendre comment les détails techniques des décisions affectent les intentions des parties prenantes, conduit à la nécessité d'explorer et d'analyser la variabilité à un niveau supérieur d'abstraction. Plusieurs typologies de variabilité ont été proposées dans cette optique. Par exemple, Salifu et al. (Salifu, 2007) utilisent les descriptions de problèmes pour représenter et analyser la variabilité dans les logiciels sensibles au contexte ; ce travail identifie le lien entre les besoins et le contexte comme une étape fondamentale pour concevoir des systèmes sensibles au contexte. Ils proposent quatre dimensions à considérer pendant l'établissement de la variabilité contextuelle et les préoccupations liées aux exigences de qualité, les phénomènes physiques, l'application d'un processus de prise de décision aux besoins de qualité et l'identification des catégories connues de problèmes. (Bashmann et al., 2001) distingue plusieurs sources de variation telles que les fonctions, les données, les contrôles de flux, la technologie, les objectifs qualité et l'environnement. Dans (America et al., 2003), la classification décrit les vues clients, application, fonctionnelle, conceptuelle et développement. Dans (Kuloor et al., 2003), l'identification des dimensions de variabilité des besoins est considérée comme un problème de séparation des besoins spécifiques du problème étudié alors que, dans (Halmans et al., 2003), la variabilité essentielle (la variabilité d'un point de vue utilisateur) se distingue de la variabilité technique (problèmes d'implémentation).

La variabilité d'un processus se doit de prendre en compte le contexte du projet sur lequel il s'applique. Pour cela, on parle de la sensibilité au contexte.

\section{La sensibilité au contexte}

(Dey et al., 2001) définit la notion de contexte par toute information pouvant être utilisée pour caractériser la situation d'une entité (personne, objet physique ou informatique), et, plus généralement, tout élément pouvant influencer le comportement d'une application. (Rey et al., 2002) propose plusieurs axes de définitions du contexte: il n'y a pas de contexte sans contexte (i.e. le contexte doit se définir en fonction d'une finalité), le contexte est un espace d'information qui sert l'interprétation (i.e. la capture du contexte n'est pas l'objectif mais les données capturées doivent servir un but), le contexte est un espace d'information partagé par plusieurs acteurs (i.e. utilisateur et système), le contexte est un espace d'information infini et évolutif (i.e. il se construit au cours du temps).

Les modèles de contexte sont pluridisciplinaires (Bradley et al., 2005). La recherche linguistique est concernée par l'analyse du contexte d'utilisation des signes (mots) dans un langage. (Bunt, 1997) définit cinq types de contexte pour les aspects communication, qui sont respectivement : linguistique (lié au matériel linguistique), sémantique (lié à la description du domaine), physique (lié à la description de l'environnement dans lequel l'action ou l'interaction se produit), 
social (lié à la situation interactive qui se produit entre acteurs) et cognitif (lié aux intentions des participants, leurs évolutions dépendant de la perception, la production, l'évaluation et l'exécution).

Le contexte est également formalisé selon des modèles mathématiques. Par exemple, (Coutaz et al., 2002) propose un modèle cumulatif où le contexte (Ctx) est une agrégation temporaire de situations. Une situation est un descripteur d'état pour un utilisateur $(U)$ exécutant une tâche $(T)$ à un certain moment $(t)$. Le modèle est décrit par la formule suivante:

$$
\left.\operatorname{Ctx}(U, T, t)=\bigcup_{n=1}^{m} \text { (Situation }\left(U, T, t_{n}\right)\right)
$$

Dans le domaine des systèmes d'information, le contexte est représenté comme un modèle ou une ontologie. Par exemple, (Gu et al., 2004) suggère une vision plus détaillée du contexte en décrivant un modèle de contexte formel basé sur une ontologie pour les environnements intelligents. Cette typologie du contexte définit un vocabulaire pour représenter la connaissance sur le contexte dans ce champ d'application particulier. Elle inclut deux niveaux : une ontologie de haut niveau capturant la connaissance du contexte général et les ontologies spécifiques aux domaines détaillant les concepts de base dans un domaine donné. Les auteurs proposent également une manière de modéliser la classification du contexte, les dépendances entre les éléments de ce contexte et sa qualité.

Le terme 'Context-awareness' (sensibilité au contexte) vient des travaux sur l'informatique pervasive, ou informatique ubiquitaire. Ces systèmes utilisent les modifications de l'environnement dans les systèmes informatiques. Même si c'est un terme d'origine informatique, il a également été appliqué à la théorie des entreprises dans le domaine de la gestion des processus métiers (Rosemann et al., 2006). C'est dans (Schilit et al., 1994) que l'on trouve la première apparition de ce terme qui ne concerne alors que des variables comme la localisation, l'identité des personnes proches et des objets ainsi que les changements dans ces objets. Plusieurs définitions existent, comme (Dey et al., 2001) pour qui un système est sensible au contexte s'il utilise le contexte pour offrir des informations ou des services pertinents pour l'utilisateur alors que (Salber et al., 1998) définit la sensibilité au contexte comme étant la meilleure capacité d'un système à agir en temps réel avec des données provenant du contexte. Plus en rapport avec notre étude, les modèles de contexte sont également proposés pour la réingénierie des processus métiers (Bessai et al., 2008), l'informatique (Bradley et al., 2005), la sélection de services (Kirsh Pinheiro et al., 2008) et la prise de décision en situation militaire (Rosen et al., 2008), (Drury et al., 2008). Dans ces derniers cas, le modèle de contexte est perçu comme une manière d'analyser une situation donnée afin de guider une exécution de tâches. Ainsi, les modèles de contexte sont principalement utilisés pour résoudre le problème de manque de flexibilité et d'adaptabilité au sein des processus. 
7 Revue Ingénierie des Systèmes d'information.

\section{Variabilité des processus téléologiques due à la sensibilité au contexte}

Nous avons mentionné que les processus téléologiques contiennent des étapes de prise de décision. Ces décisions peuvent porter sur les modèles à utiliser, actions à effectuer, scénarios possibles etc. Ce sont notamment les décisions prises au cours de l'exécution des processus téléologiques qui permettent de les adapter au contexte et, par conséquent, d'assurer leur variabilité. Chaque fois qu'un ingénieur doit choisir entre plusieurs possibilités pour continuer son processus, il est confronté à un problème de prise de décision.

Un problème de prise de décision est défini par la présence d'au moins deux alternatives. Le concept d'alternative désigne l'objet de la décision. Les alternatives doivent être identifiées clairement. Ces alternatives sont comparées entre elles selon un ou plusieurs critères. Les critères représentent des informations qui permettent d'évaluer et de comparer les alternatives. Ils peuvent être de nature différente : les caractéristiques intrinsèques des objets de décision, avis des parties prenantes, conséquences potentielles des alternatives etc. Le problème de prise de décision comporte également la problématique de décision qui représente la solution cible et peut représenter : un choix (sélection d'un sous-ensemble d'alternatives potentielles ou une alternative, le cas échéant) ; un rangement (ordonnancement des alternatives potentielles) ou bien un tri (affectation des alternatives potentielles à un ensemble de classes prédéfinies). De cette manière, le problème de prise de décision contient trois éléments de base : la problématique, les alternatives et les critères (Roy, 2005).

Dans le cadre de notre approche chaque étape de prise de décision dans les processus téléologiques est considérée comme un problème de prise de décision. Plus précisément, la problématique est le choix, les alternatives sont des variantes potentiellement disponibles dans un processus sur chacun des points de variation et les critères sont des indicateurs de contexte.

\subsection{Typologie d'indicateurs}

Les processus téléologiques contiennent des points de variation où l'ingénieur doit prendre une décision entre plusieurs alternatives. Il se retrouve donc confronté à un problème de choix. L'évaluation de ce problème, et sa résolution, peuvent être envisagées de plusieurs manières. Par exemple, la méthode I* préconise l'utilisation de la notion d'exigences non fonctionnelles en utilisant les 'soft goals' identifiés comme des critères d'évaluation. Les alternatives contribuent alors à différents degrés de satisfaction de ces buts (van Lamswerde, 2009).

Dans notre approche, pour résoudre le problème de prise de décision de notre ingénieur, nous proposons d'utiliser en priorité le contexte du projet. Ce contexte est composé d'un ensemble d'indicateurs permettant de le caractériser (figure 1). 
Processus téléologique et variabilité : Utilisation de la sensibilité au contexte 8

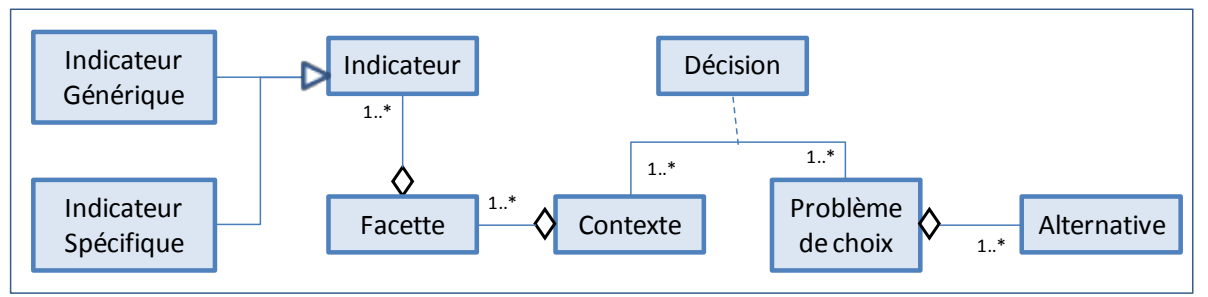

Figure 1. Sensibilité au contexte des processus téléologiques

Afin d'établir cette typologie, nous avons défini les indicateurs couvrant les aspects essentiels de l'ingénierie des projets de SI. Des indicateurs découlant des exigences non fonctionnelles peuvent également être intégrés dans cette typologie pour la rendre plus exhaustive, ce qui fera l'objet d'un futur travail sur les indicateurs.

Nous distinguons ici deux types d'indicateurs: génériques et spécifiques. Les premiers sont communs à la plupart des méta-modèles de processus alors que les autres varient d'un méta-modèle de processus à l'autre.

Indicateurs génériques. Nous avons établi une liste d'indicateurs génériques en nous basant sur le travail de (Kornyshova et al., 2007). Les auteurs proposent une typologie d'indicateurs de projet dans le but de guider la recherche et la priorisation de composants de méthodes. Cette proposition est basée sur les travaux de (Mirbel et al., 2006), (Van Slooten et al., 1996) et (Kornyshova et al., 2007). Elle inclut quatre facettes :

- la facette organisationnelle,

- la facette humaine,

- la facette du domaine d'application et

- la facette de la stratégie de développement.

La facette organisationnelle (Table 1) met en valeur les aspects organisationnels du développement de projets de système d'information. Par exemple, l'indicateur Implication de l'équipe de management représente l'investissement de l'équipe de direction dans le projet. Les valeurs possibles pour cet indicateur sont 'Faible', 'Normale' et 'Elevée' (i.e. une valeur élevée signifie un grand investissement). 
9 Revue Ingénierie des Systèmes d'information.

\begin{tabular}{|l|l|}
\hline Indicateur & Domaine de valeur \\
\hline $\begin{array}{l}\text { Implication de l'équipe de } \\
\text { management }\end{array}$ & \{Faible, normal, élevé\} \\
\hline Importance & $\{$ Faible, normal, élevé $\}$ \\
\hline Impact & $\{$ Faible, normal, élevé\} \\
\hline Urgence & $\{$ Faible, normal, élevé $\}$ \\
\hline Limitation des ressources & $\{$ Faible, normal, élevé\} \\
\hline $\begin{array}{l}\text { Nature des ressources } \\
\text { limitées }\end{array}$ & $\begin{array}{l}\{\text { ressources financières, ressources temporelles, } \\
\text { ressources informatiques }\}\end{array}$ \\
\hline Taille & $\{$ Faible, normal, élevé\} \\
\hline Coût & $\{$ Faible, normal, élevé $\}$ \\
\hline Niveau d'innovation & $\{$ Faible, normal, élevé $\}$ \\
\hline Nature de l'innovation & $\{$ Innovation métier, innovation technologique $\}$ \\
\hline
\end{tabular}

Table 1. Valeurs de la facette organisationnelle.

La facette humaine (Table 2) décrit les indicateurs des personnes impliquées dans le développement du projet. Par exemple, l'indicateur du type de participation des utilisateurs représente les différentes manières qu'ont les utilisateurs de participer au projet, ses valeurs pouvant être 'Réel' ou 'Virtuel'.

\begin{tabular}{|l|l|}
\hline Indicateur & Domaine de valeur \\
\hline Résistance et Conflit & $\{$ Faible, normal, élevé $\}$ \\
\hline Degré d'expertise & $\{$ Faible, normal, élevé $\}$ \\
\hline Rôle de l'expert & $\begin{array}{l}\{\text { Testeur, Développeur, Concepteur, } \\
\text { Analyste }\}\end{array}$ \\
\hline Clarté et stabilité & $\{$ Faible, normal, élevé $\}$ \\
\hline Type de participation des utilisateurs & $\{$ Réel, Virtuel $\}$ \\
\hline Nombre d'utilisateurs & Nombre \\
\hline
\end{tabular}

\section{Table 2. Valeurs de la facette humaine}

La facette du domaine d'application (Table 3) inclut les indicateurs caractérisant le domaine du projet de système d'information. Par exemple, l'indicateur Type de l'application permet de spécifier la structure organisationnelle du projet. Il peut prendre les valeurs 'Application Intra-organisation', 'Application Inter-organisation' ou encore 'Application Organisation-Client'. 
Processus téléologique et variabilité : Utilisation de la sensibilité au contexte $\mathbf{1 0}$

\begin{tabular}{|l|l|}
\hline Indicateur & Domaine de valeur \\
\hline Degré de formalisation & $\{$ Faible, normal, élevé\} \\
\hline Degré de relations & $\{$ Faible, normal, élevé\} \\
\hline Degré de dépendances & $\{$ Faible, normal, élevé\} \\
\hline Complexité & $\{$ Faible, normal, élevé\} \\
\hline Type de l'application & $\begin{array}{l}\text { \{Application intra-organisation, Application inter- } \\
\text { organisation, Application organisation-client }\}\end{array}$ \\
\hline $\begin{array}{l}\text { Technologie de } \\
\text { l'application }\end{array}$ & $\begin{array}{l}\text { \{l'application à développer inclut une base de données, } \\
\text { l'application à développer est distribuée, l'application à } \\
\text { développer inclut une interface utilisateur graphique\} }\end{array}$ \\
\hline Granularité du projet & $\begin{array}{l}\text { \{système unique, sous-projets orientés-système, sous- } \\
\text { projets orientés-processus, sous-projets hybrides }\}\end{array}$ \\
\hline Répétition & \{Faible, normal, élevé\} \\
\hline Variabilité & $\{$ Faible, normal, élevé\} \\
\hline Artéfacts variables & $\begin{array}{l}\text { \{organisationnel, humain, domaine d'application, } \\
\text { stratégie de développement }\end{array}$ \\
\hline
\end{tabular}

\section{Table 3. Valeurs de la facette du domaine d'application}

La facette de la stratégie de développement (Table 4) permet de spécifier différentes informations. Par exemple, l'indicateur représentant l'origine du système représente l'origine des éléments réutilisés pouvant être soit du code, soit du domaine fonctionnel, soit encore des interfaces.

\begin{tabular}{|l|l|}
\hline Indicateur & Domaine de valeur \\
\hline Origine du système & $\begin{array}{l}\{\text { réutilisation du code, réutilisation de domaine } \\
\text { fonctionnel, réutilisation d'interface }\}\end{array}$ \\
\hline Organisation de projet & $\{$ standard, adaptée $\}$ \\
\hline Stratégie de développement & $\begin{array}{l}\{\text { d'outsourcing, itérative, de prototypage, } \\
\text { orientée phases, orientée composants }\}\end{array}$ \\
\hline Stratégie de réalisation & $\begin{array}{l}\{\text { en une fois, de façon incrémentale, de façon } \\
\text { concurrentielle, avec overlapping }\}\end{array}$ \\
\hline Stratégie de livraison & $\begin{array}{l}\{\text { en une fois, de façon incrémentale, de façon } \\
\text { évolutive }\}\end{array}$ \\
\hline Traçabilité & $\{$ Faible, forte $\}$ \\
\hline Nombre d'objectifs & $\{$ objectif unique, multiples objectifs $\}$ \\
\hline
\end{tabular}

Table 4. Valeurs de la facette de la stratégie de développement 
11 Revue Ingénierie des Systèmes d'information.

Indicateurs spécifiques. Leur identification est basée sur la description de la méthode. L'ingénieur de méthodes les définit en analysant différents aspects organisés en quatre facettes: intentionnelle, satisfactionnelle, décisionnelle et interne, comme dans (Harmsen, 1997).

- La facette intentionnelle concerne les intentions de la méthode.

- La facette satisfactionnelle indique le degré de satisfaction que l'ingénieur a au sujet des résultats de l'application de la méthode.

- La facette décisionnelle représente les processus de prise de décision de la méthode.

- La facette interne concerne les critères connus associés à une gestion de projet spécifique.

Selon le méta-modèle de processus utilisé dans la méthode, les indicateurs qui seront définis dans chacune de ces quatre facettes pourront être différents puisqu'ils seront basés sur les concepts et les règles de ce méta-modèle de processus spécifique.

Typologie des indicateurs. Les indicateurs définis ci-dessus peuvent être représentés au sein d'une même typologie. La figure suivante montre la typologie des indicateurs proposée.

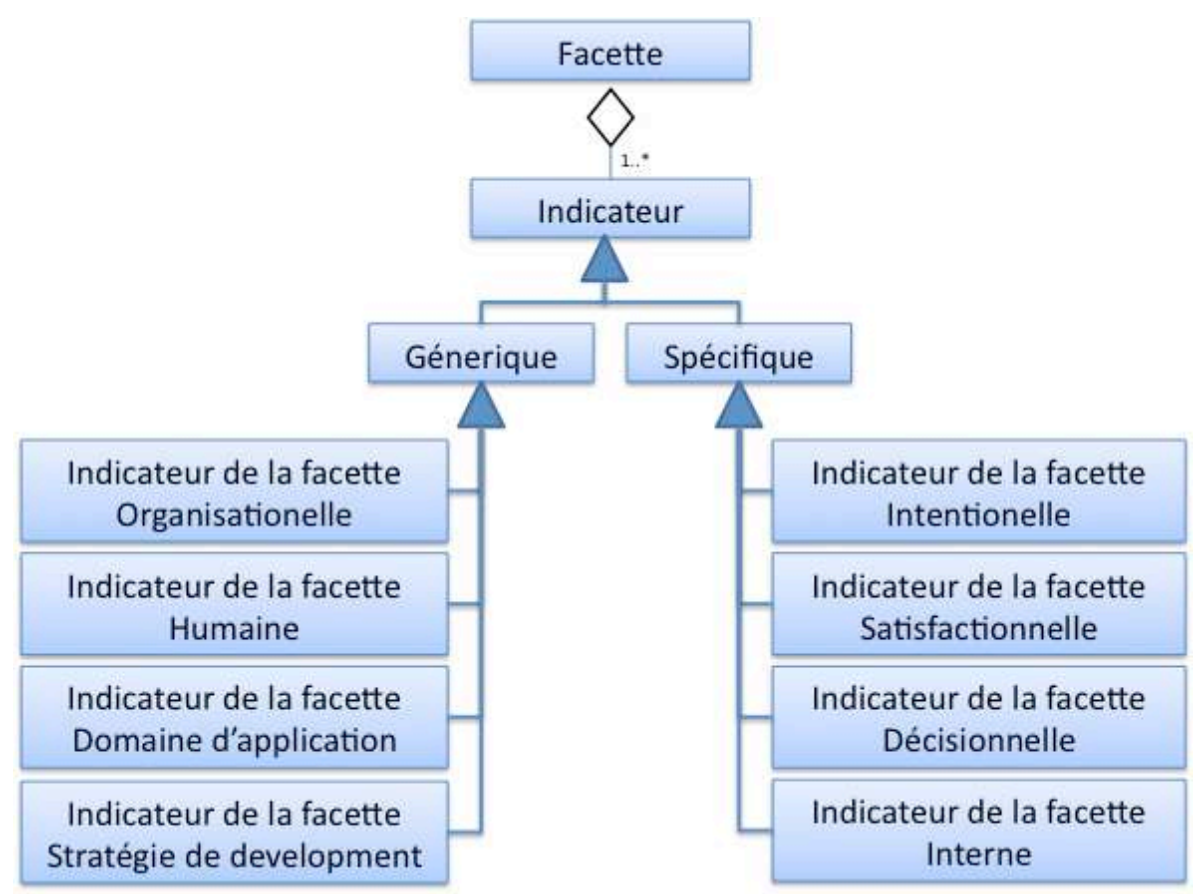


Figure 2. Typologie des indicateurs

Cette typologie indique les principaux indicateurs qui peuvent être définis dans une situation donnée. Elle peut bien entendu être complétée si de nouveaux indicateurs apparaissent, notamment ceux qui découleraient d'une étude plus approfondie des besoins non fonctionnels. Il pourrait également être envisageable de définir les différents liens pouvant exister entre les différents indicateurs ou les différentes facettes, ce qui n'a pas encore été fait dans le travail existant.

Les indicateurs définis dans cette typologie ont des valeurs assez informelles mais qui n'empêchent pas l'utilisation de méthodes d'aide à la décision multicritères. Ces valeurs peuvent par exemple être remplacées par des valeurs numériques (les valeurs 'Faible', 'Normal', 'Elevé' peuvent être remplacées par les valeurs '1', '2' et '3'). Une fois plus formelles, les valeurs des différents indicateurs peuvent être agrégées en une valeur générale en utilisant certaines techniques (Roy, 1996) (Keeney, 1993) (Keeney 1999) pour pouvoir être utilisées comme un critère unique sur lequel différentes fonctions pourront être utilisées (somme, maximum, minimum, moyenne, somme pondérée...).

\subsection{Processus d'utilisation}

Le processus d'utilisation de cette typologie est appelé IG ('Indicator-based Guidance' ou Guidage basé sur les Indicateurs) et est illustré de manière simplifiée dans la figure 3 suivante.

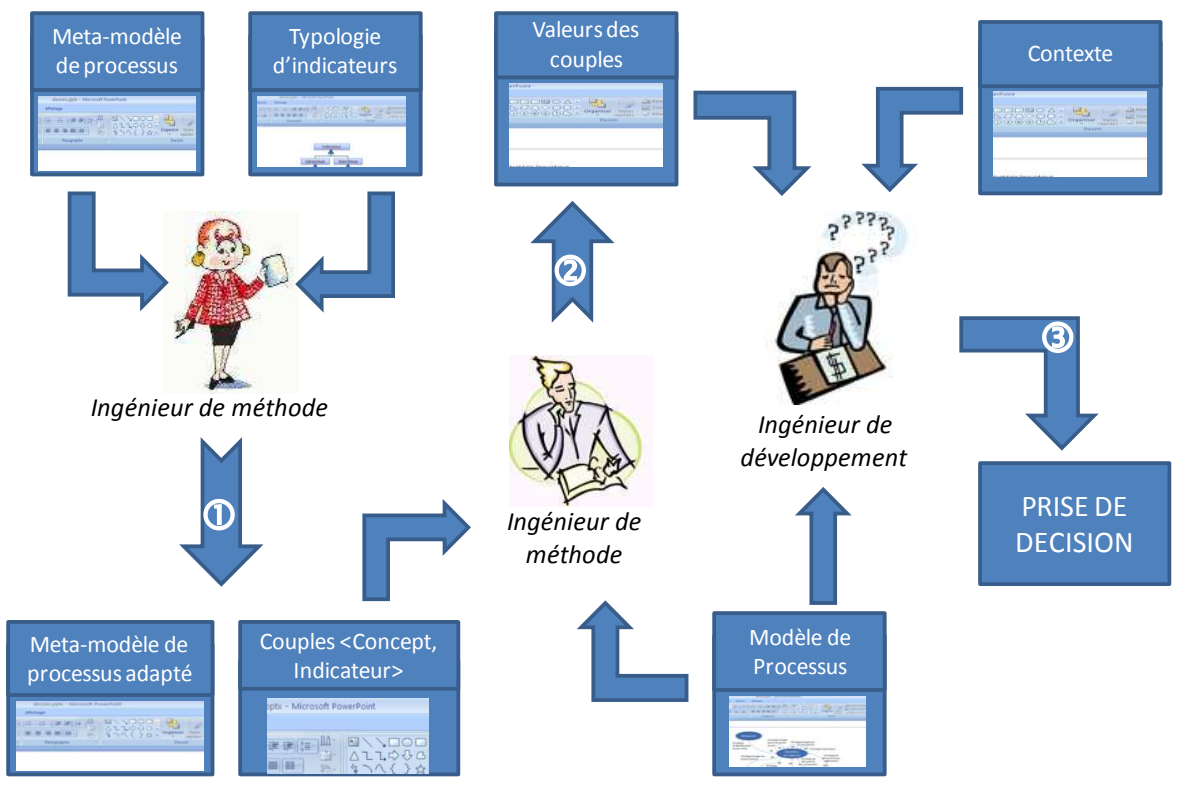

Figure 3. Processus $I G$ 
Le processus IG s'effectue à deux niveaux différents : au niveau modèle et au niveau instance. Le premier niveau fait l'objet de la phase préparatoire où l'ingénieur de méthodes va intégrer le concept d'indicateurs dans le méta-modèle de processus que l'ingénieur de développement utilise (niveau modèle - flèche (1) à la figure 3). Le deuxième niveau correspond aux étapes d'utilisation de la typologie d'indicateurs, tout d'abord pour donner des valeurs aux indicateurs (niveau modèle flèche (2) à la figure 3) avant la phase d'utilisation proprement dite où l'ingénieur de développement va se servir des valeurs d'indicateurs et de la valeur de son contexte de projet pour prendre ses décisions (niveau instance - flèche (3) à la figure 3).

Ces divers processus sont représentés avec le méta-modèle MAP (utilisé dans cet article pour illustrer la proposition et qui est expliqué dans la section suivante) dans les figures 4 et 5 .

\section{Processus IG de l'ingénieur de méthodes}

L'ingénieur de méthodes est l'acteur qui va utiliser le processus IG au niveau modèle. C'est à cette étape que le méta-modèle de processus utilisé va être étudié. Lorsque cette partie du processus sera terminée, (1) le concept d'indicateur aura été intégré dans le méta-modèle et (2) les concepts impactés par les indicateurs auront été déterminés.

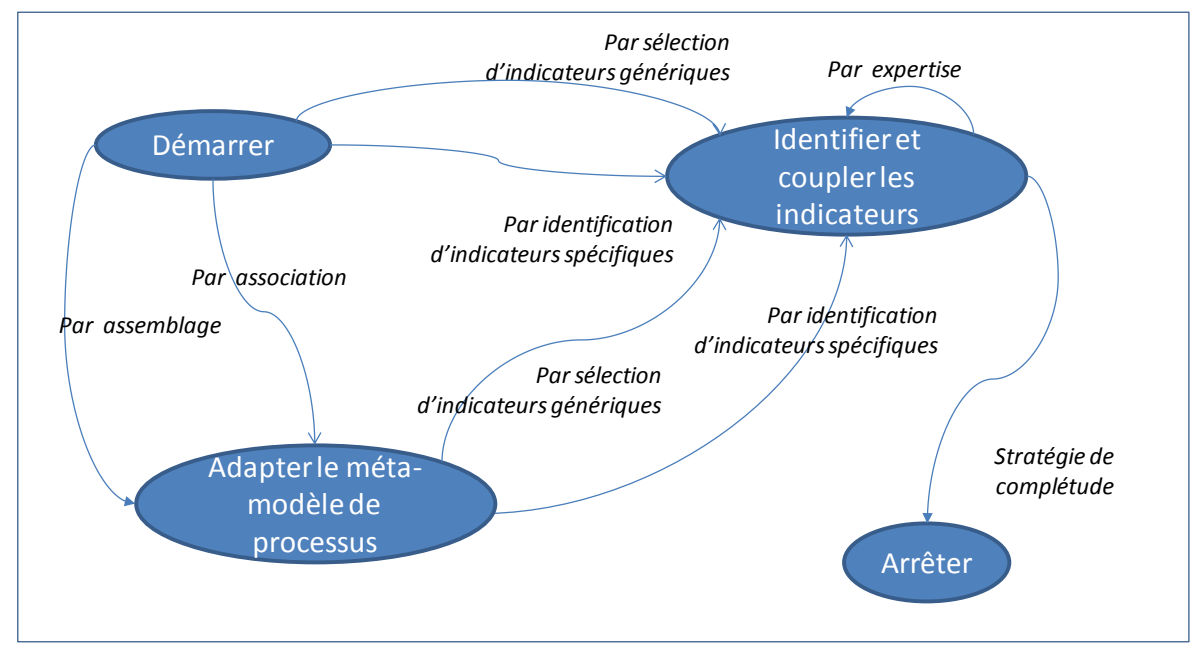

Figure 4. Processus IG de l'ingénieur de méthodes

Au niveau du méta-modèle de processus, l'ingénieur de méthodes doit tout d'abord y intégrer le concept général d'indicateur pour déterminer quels sont les concepts pour lesquels le fait de rattacher des indicateurs de contexte a un sens. Cette intégration se fait selon le principe de l'assemblage de composants de méthode défini par (Ralyté, 2001). Cette approche distingue deux possibilités d'intégration : si le concept d'indicateur est complètement absent du méta-modèle de processus 
choisi, alors l'intégration de ce concept se fera grâce à une simple association avec les concepts déjà présents (stratégie Par association), cependant, si la sémantique des concepts du méta-modèle de processus se chevauche avec celle du concept d'indicateur que l'on veut intégrer, il faut alors modifier le méta-modèle de processus pour pouvoir effectuer l'intégration en conservant la cohérence du métamodèle (stratégie Par assemblage). Il est également possible que le concept d'indicateur, avec la même sémantique que celui qui nous intéresse, soit déjà intégré dans le méta-modèle de processus, dans ce cas, cette étape d'intégration ne sera pas utile et l'ingénieur de méthodes pourra directement passer à l'utilisation de la typologie d'indicateurs.

Il est donc ensuite nécessaire d'identifier quels sont les différents indicateurs génériques s'appliquant à chacun des concepts du méta-modèle de processus. Cette étape se réalise en utilisant la stratégie par sélection d'indicateurs génériques. C'est en effet en étudiant le méta-modèle de processus que l'ingénieur de méthodes va pouvoir identifier les différents couples (indicateur générique, concept du métamodèle) qui seront utiles à l'ingénieur de développement.

La stratégie par identification d'indicateurs spécifiques permettra à l'ingénieur de méthodes de déterminer quels sont les indicateurs spécifiques au méta-modèle de processus qu'il peut ajouter dans son intégration. De même que pour la stratégie précédente, il lui faudra former des couples (indicateur spécifique, concept du métamodèle) pour garantir la cohérence de l'utilisation des indicateurs.

L'ingénieur de méthodes a également la possibilité d'effectuer des ajustements sur les indicateurs utilisés pendant leur intégration. La stratégie par expertise lui permettra d'ajouter ou de supprimer un indicateur ou d'en modifier un déjà sélectionné.

\section{Processus IG de l'ingénieur de développement}

L'ingénieur de développement est l'acteur qui va utiliser le processus pour prendre des décisions. Il va se servir des modifications effectuées par l'ingénieur de méthodes au niveau modèle pour utiliser au mieux les indicateurs et faire ses choix à bon escient. Pour chacune des décisions que l'ingénieur aura à prendre, le processus IG (1) lui permettra d'évaluer les valeurs des indicateurs pertinents et (2) lui proposera différentes manières de résoudre son problème de prise de décision à l'aide de ces valeurs d'indicateurs. Ce processus est illustré à la figure 5 .

Au niveau instance, l'ingénieur de développement doit tout d'abord évaluer la situation. La stratégie Par expertise du méta-modèle de processus lui permettra d'affecter une valeur à chaque couple (indicateur, concept) défini par l'ingénieur de méthodes. Cette étape n'est à effectuer qu'une seule fois par méta-modèle de processus. En effet, si le même méta-modèle est utilisé lors d'une autre réalisation du processus IG, il suffira à l'ingénieur de développement de reprendre les valeurs définies lors d'une précédente évaluation. La stratégie Par expertise des 
15 Revue Ingénierie des Systèmes d'information.

caractéristiques du projet, quant à elle, permettra à l'ingénieur de développement de définir certaines caractéristiques du projet concerné par son étude.

Ensuite, l'ingénieur de déveoppement peut utiliser ces valeurs de caractérisation du contexte et des indicateurs pour prendre des décisions. L'utilisation de la stratégie par application de méthodes de choix lui permet d'utiliser des techniques de décision mono ou multicritères dans ce sens.

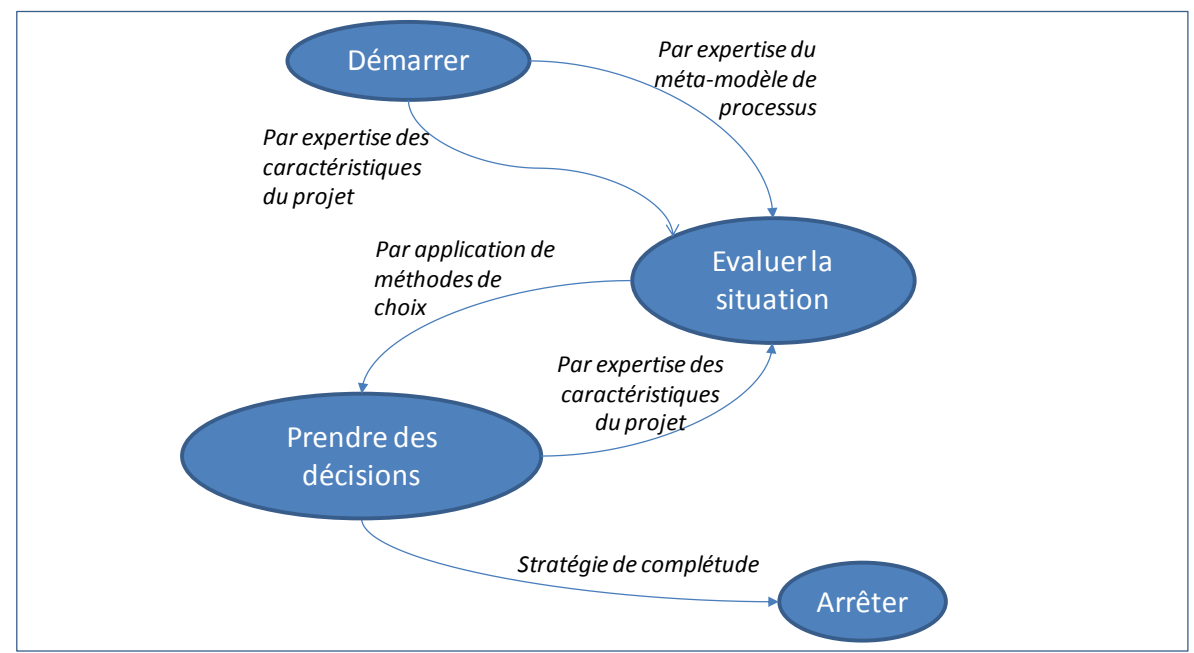

Figure 5. Processus IG de l'ingénieur de développement

Chaque décision prise peut ensuite être prise en compte par l'ingénieur pour effectuer une réévaluation de la situation. La stratégie Par expertise des caractéristiques du projet permet ici à l'ingénieur de développement de réévaluer le contexte de son projet avant la prochaine prise de décision.

Ce processus d'utilisation s'arrête lorsque le processus de développement ne comporte plus aucun point de prise de décision.

\section{Application du processus de sensibilisation au méta-modèle de processus MAP}

Le méta-modèle MAP (Rolland et al., 1999) (Rolland, 2007) est un système de représentation initialement développé pour représenter les modèles de processus en termes intentionnels. La plupart des modèles n'intègrent pas réellement le concept d'intention, se concentrant sur la manière dont le processus est exécuté et externalisant dans l'intention ce que le processus est destiné à accomplir (Dietz, 2004). En revanche, les modèles de processus intentionnels se concentrent sur ce que le processus essaie d'atteindre, offrant ainsi la raison d'être du processus, à savoir pourquoi le processus est exécuté. MAP permet de spécifier les processus 
d'une manière flexible en mettant l'accent sur les intentions de processus et sur les différentes manières d'atteindre chacune de ces intentions.

Comme le montre l'exemple donné en figure 6, une carte est présentée comme un diagramme dont les nœuds sont les intentions et les arcs les stratégies. Cette carte représente le processus de l'approche Crews-L'écritoire et est décrite en détails dans (Ralyté et al., 1999).

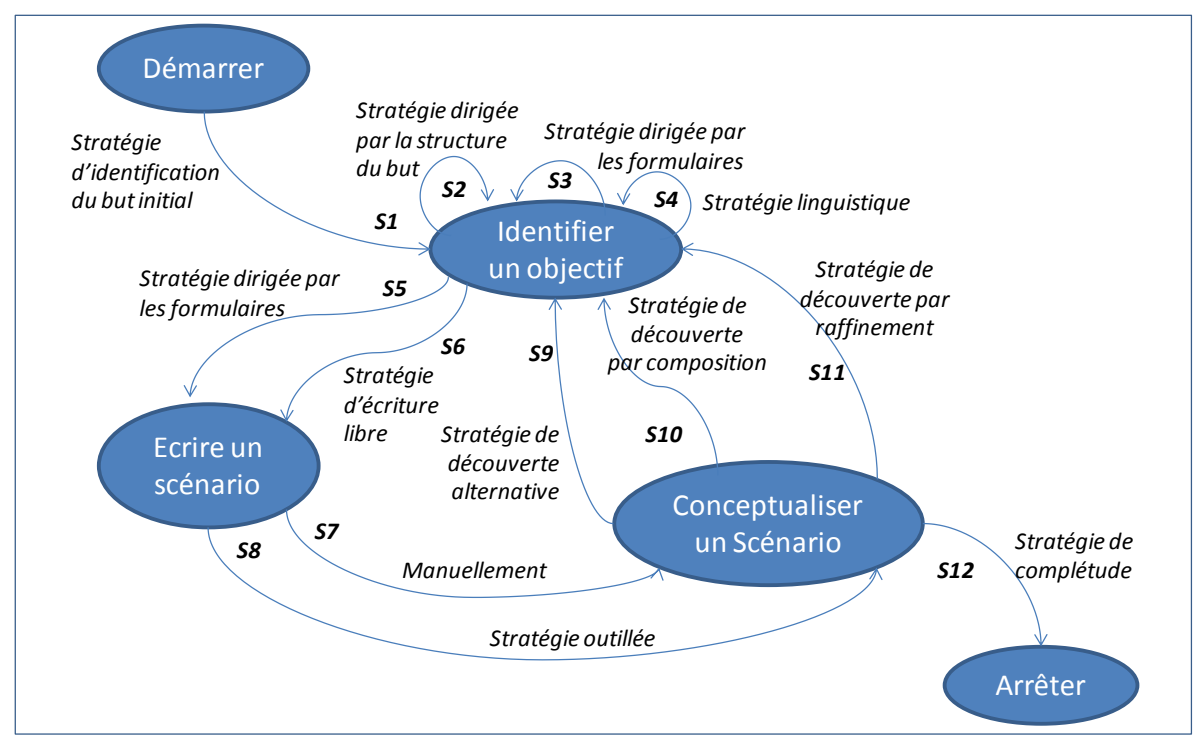

Figure 6. Un exemple de carte : celle de l'approche Crews-L'écritoire

Les arcs orientés montrent quelles sont les intentions qui peuvent précéder ou suivre les autres. Un arc entre dans un nœud si sa stratégie associée peut être utilisée pour réaliser l'intention cible (le nœud). Comme il peut y avoir plusieurs arcs entrant dans un nœud, cela représente tous les moyens pour réaliser une intention.

Le but de cette modélisation est de décrire ce que l'entreprise veut réaliser ou éviter. Raisonner sur les objectifs facilite la compréhension des problèmes et la communication sur les aspects essentiels (quoi et pourquoi, au lieu de qui, quand, où et comment) (Nurcan, 2004).

Le méta-modèle de processus MAP est défini à la figure 7. Le concept principal de ce méta-modèle est celui de Section. Une carte est composée d'un ensemble de sections. Une section est associée avec deux intentions, l'intention source et l'intention cible, reliées entre elles par une stratégie. Elle intègre les connaissances correspondant à une étape spécifique du processus pour réaliser une intention (l'intention cible) à partir d'une situation spécifique (l'intention source) en suivant une technique particulière (la stratégie). L'intention est un objectif qui peut être atteint par l'exécution d'un processus. Chaque carte comprend deux intentions 
17 Revue Ingénierie des Systèmes d'information.

prédéfinies : "Démarrer" et "Arrêter" (début et fin du processus). Une manière spécifique de réaliser une intention est capturée dans une section de la carte, sachant que toutes les sections ayant les mêmes sources et cibles permettent de représenter les différentes stratégies qui peuvent être utilisées pour réaliser cette intention cible. De la même façon, il peut y avoir plusieurs sections avec la même intention source, mais des intentions cibles différentes. Cela permet de montrer toutes les intentions qui peuvent être atteintes après la réalisation de l'intention source.

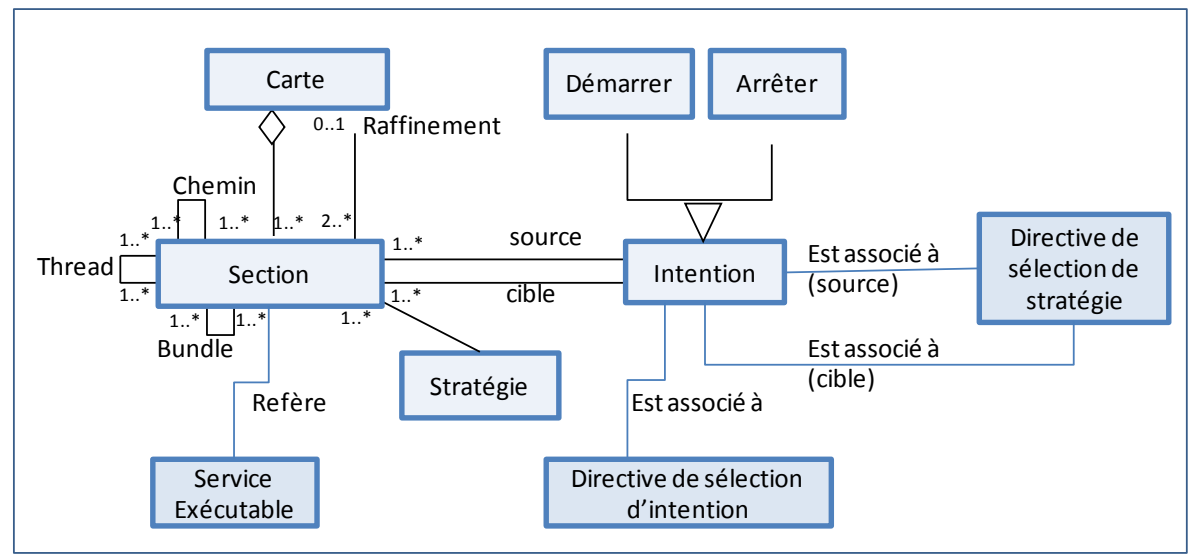

Figure 7. Le méta-modèle de processus MAP

Les sections sont à un niveau d'abstraction qui permet de capturer la variabilité $\mathrm{du}$ processus puisque chaque section correspond à l'application d'un service exécutable. Comme dans (Rolland et al., 2007b) et (Rolland et al., 2007c), notre point de vue est qu'une section représente une caractéristique visible du processus et une abstraction d'un flux cohérent d'activités exprimées de manière intentionnelle. Chaque section de la carte identifie ce que nous pouvons appeler une variante.

Les variantes (sections) d'une carte sont liées les unes aux autres par trois types de relations (thread, bundle et chemin) qui génèrent des multi-thread et des multichemins dans une carte (Prakash et al., 2006).

- Un thread montre la possibilité d'atteindre la même intention cible, à partir d'une intention source unique, de plusieurs façons. Chacune de ces techniques est exprimée dans la carte comme une section spécifique. L'ensemble des sections participant à une telle relation est appelée un multi-thread. Cette relation exprime directement la variabilité associée à un processus multi-objectifs puisque chaque but est intégré dans les différentes stratégies permettant d'atteindre l'intention cible commune (Rolland, 2007).

- Un chemin établit une relation de précédence entre les sections. Pour qu'une section puisse succéder à une autre, son intention source doit être l'intention cible de la section précédente. Une carte contient un nombre fini de chemins allant de l'intention Start à l'intention Stop. Chacun d'eux est un modèle de processus. En ce 
sens la carte peut être considérée comme un multi-modèle. En naviguant dans la carte l'ingénieur de développement sélectionne le chemin et construit dynamiquement le modèle de processus qu'il applique. Aucun chemin n'est recommandé à priori. Au contraire c'est en fonction des situations qu'il rencontre que l'ingénieur choisira un chemin plutôt qu'un autre. Avec les relations de thread et de chemins, un but peut être atteint par plusieurs combinaisons de sections. Un tel ensemble est appelé un multi-chemins et peut contenir plusieurs threads. La relation multi-chemins est une autre façon de représenter la perspective pluri-objectifs du modèle de processus (Rolland, 2007).

- Un bundle indique la possibilité pour plusieurs sections, ayant les mêmes intentions sources et cibles, d'être mutuellement exclusives.

Une relation de raffinement montre qu'une section de la carte peut être raffinée par une autre carte. C'est un mécanisme d'abstraction par lequel un assemblage complexe de sections au niveau $i+1$ est considéré comme une section unique au niveau $i$. Cela introduit le concept de niveaux dans la représentation du processus puisque cela implique que chaque carte peut être représentée comme une hiérarchie de cartes. Cette hiérarchie permet d'obtenir une carte de haut niveau du processus ainsi que des cartes affinant ses sections particulièrement stratégiques, et ceci de manière récursive.

Chaque section est reliée à un service exécutable. Il fournit un moyen opérationnel de réaliser l'intention. Il implique la transformation du produit en cours d'élaboration par l'exécution d'un service (qui peut être une directive, un workflow, un algorithme, etc.).

Il existe deux directives associées à chaque section. La première est la directive de sélection d'intention (DSI). Elle définit l'ensemble des intentions qui peuvent être obtenues à l'étape suivante (lorsque la section aura été réalisée) et propose des arguments de sélection. La seconde est appelé directive de sélection de stratégie (DSS). Elle complète la première car elle détermine toutes les stratégies pouvant être utilisées pour atteindre le but visé (identifiée avec la directive précédente) ainsi que les arguments permettant à l'ingénieur de faire son choix.

\section{Première phase : Au niveau modèle, par l'ingénieur de méthodes}

La première étape consiste en l'intégration du concept d'indicateur dans le métamodèle de processus d'origine puisque celui-ci n'a pas encore été adapté.

Cette étape utilise ici une approche par assemblage pour effectuer cette intégration puisque qu'il y a un chevauchement entre le concept d'indicateur et deux concepts du méta-modèle MAP : la directive de sélection d'intention et la directive de sélection de stratégie. Ces deux concepts permettent de donner des arguments concernant la navigation dans la carte, ceci en langage naturel. Elles peuvent cependant être traduites par des indicateurs qui prendront leur valeur dans les arguments qui auraient été placés dans ces concepts, applicables aux concepts 
19 Revue Ingénierie des Systèmes d'information.

d'Intention et de Stratégie. Par exemple, si l'argument est 'l'auteur du scénario doit être un expert en écriture de scénario', cela peut se traduire par l'utilisation de l'indicateur Rôle de l'expert avec une valeur 'Analyste'. De la même manière, l'argument 'l'auteur écrit le scénario en prose libre' implique une valeur 'Faible' pour l'indicateur du Degré de formalisation alors que l'argument 'l'auteur écrit le scénario en remplissant un formulaire linguistique' impliquera une valeur 'Elevée' du même indicateur.

Le concept d'indicateur peut donc se rattacher à quatre concepts du méta-modèle MAP : l'Intention, la Stratégie, la Section et la Carte elle-même, ainsi qu'il est indiqué dans la figure suivante.

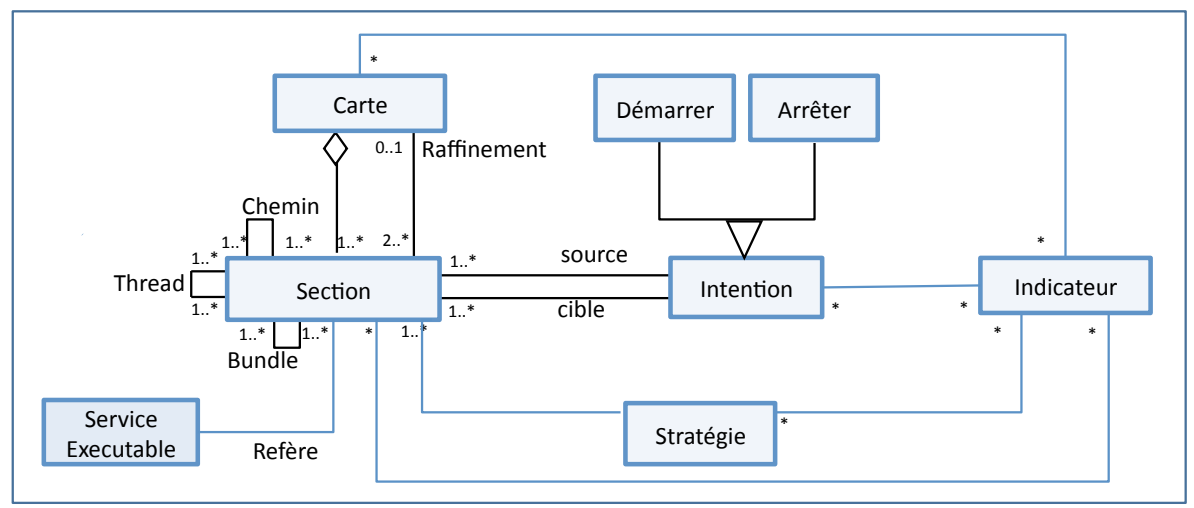

Figure 8. Le méta-modèle de processus MAP sensible au contexte.

La suite de cette étape est de définir les couples (indicateur, concept). Nous allons illustrer notre propos avec l'indicateur Degré d'expertise ((Deneckere et al., 2010) propose une illustration plus complète de l'application d'indicateurs au métamodèle MAP). Cet indicateur va essentiellement se définir pour les stratégies. En effet, le fait d'effectuer une tâche d'une certaine manière va correspondre à un niveau d'expertise minimal. Par relation, la Section et la Carte également vont pouvoir être définies pour un niveau d'expertise particulier. Les couples obtenus sont donc ceux illustrés à la table 5 .

\begin{tabular}{|c|c|}
\hline Indicateur générique & Concept \\
\hline Degré d'expertise & Stratégie \\
\hline Degré d'expertise & Section \\
\hline Degré d'expertise & Carte \\
\hline
\end{tabular}

Table 5. Couples identifiés pour les indicateurs génériques

La deuxième étape permet d'identifier les indicateurs spécifiques au métamodèle MAP. La typologie des indicateurs identifie quatre facettes différentes pour les indicateurs spécifiques (intentionnelle, satisfactionnelle, décisionnelle et interne). 
La facette intentionnelle est représentée dans le méta-modèle MAP par l'intention cible d'une section. Cette intention représente le prochain but à réaliser, le prochain objectif à atteindre pour pouvoir continuer la navigation dans la carte. Nous pouvons donc identifier l'indicateur Intention dans cette facette, comme il est indiqué dans la table 6.

\begin{tabular}{|l|l|}
\hline Indicateur & Domaine de valeur \\
\hline Intention & Texte \\
\hline
\end{tabular}

Table 6. Valeurs de la facette intentionnelle

Nous pouvons identifier deux indicateurs pour la typologie applicable aux cartes, représentant des indicateurs dynamiques, et appartenant à la facette satisfactionnelle. Ces indicateurs, évalués à la volée pendant l'exécution du processus, tiennent compte de la situation en évolution constante du produit en cours de construction. Par exemple, si l'exécution d'une section ne réalise pas complètement l'intention cible, il sera alors nécessaire d'exécuter un cycle sur cette intention, afin de la réaliser plus complètement (Indicateur Etat de l'intention). Des indicateurs fournissent également une évaluation de la complétude de la section. Suite à la situation du produit en construction, une section peut devoir être exécutée à plusieurs reprises afin de réaliser complètement la directive (Indicateur Réalisation de la directive). La table 7 résume les deux indicateurs de la facette satisfactionnelle.

\begin{tabular}{|l|l|}
\hline Indicateur & Domaine de valeur \\
\hline Etat de l'intention & $\{$ Faible, moyen, élevé \\
\hline Réalisation de la directive & Réalisée, Faible réalisation $\}$ \\
\hline
\end{tabular}

Table 7. Valeurs de la facette satisfactionnelle

La facette décisionnelle représente les processus de prise de décision de la méthode. Dans le méta-modèle MAP, ces prises de décisions sont effectuées au niveau des stratégies à utiliser pour effectuer la suite de la navigation. L'indicateur pouvant être utilisé dans la facette décisionnelle est donc la Stratégie, comme l'indique la table 8 .

\begin{tabular}{|l|l|}
\hline Indicateur & Domaine de valeur \\
\hline Stratégie & Texte \\
\hline
\end{tabular}

Table 8. Valeurs de la facette décisionnelle

La facette interne correspond à la définition des critères connus associés à une gestion de projet spécifique dans le méta-modèle de processus. Le méta-modèle MAP ne possède pas d'indicateur appartenant à cette facette. 
La fin de la deuxième étape permet de construire les couples. Nous choisissons d'illustrer cette étape uniquement avec l'indicateur spécifique Etat de l'intention. Cet indicateur s'applique au concept de Section (la section peut être exécutée si l'intention cible n'est pas complètement réalisée). Le couple obtenu est donc celui indiqué dans la table 9.

\begin{tabular}{|c|c|}
\hline Indicateur spécifique & Concept \\
\hline Etat de l'intention & Section \\
\hline
\end{tabular}

Table 9. Couple identifié pour les indicateurs spécifiques

En résumé, dans cet exemple particulier, les couples (indicateur, concept) obtenus sont les suivants :

\begin{tabular}{|c|c|}
\hline Indicateur & Concept \\
\hline Degré d'expertise & Stratégie \\
\hline Degré d'expertise & Section \\
\hline Degré d'expertise & Carte \\
\hline Etat de l'intention & Section \\
\hline
\end{tabular}

Table 10. Couples identifiés pour l'exemple d'application

\section{Deuxième phase : Au niveau instance,par l'ingénieur de développement}

La première étape (flèche 2 de la figure 3) consiste à évaluer le contexte à l'aide des indicateurs génériques et spécifiques. L'indicateur générique choisi pour illustrer le processus est celui du Degré d'expertise. Cet indicateur s'applique tout d'abord au concept de Stratégie. Par relation, la même valeur pourra être affectée à la Section utilisant cette stratégie, sachant que le niveau d'expertise se définit avec les valeurs 'Faible', 'Moyen' et 'Elevé'. L'indicateur spécifique choisi est l'Etat de l'intention, le concept impacté est la Section et les valeurs sont 'Réalisée' ou 'Faible réalisation'.

Dans la carte de l'exemple (Figure 3), nous pouvons affecter les valeurs indiquées dans la Table 11.

\begin{tabular}{|l|c||l|c|l|}
\hline Stratégie & $\begin{array}{l}\text { Degré } \\
\text { d'expertise }\end{array}$ & Section & $\begin{array}{l}\text { Degré } \\
\text { d'expertise }\end{array}$ & Etat de l'intention \\
\hline Identification du but initial & Faible & S1 & Faible & Réalisée \\
\hline Dirigée par les formulaires & Moyen & S2 & Elevé & Faible realisation \\
\hline Dirigée par la structure du but & Elevé & S3 & Moyen & Faible realisation \\
\hline Linguistique & Moyen & S4 & Moyen & Faible realisation \\
\hline Ecriture libre & Faible & S5 & Elevé & Réalisée \\
\hline Manuellement & Elevé & S6 & Faible & Réalisée \\
\hline Outillée & Moyen & S7 & Moyen & Réalisée \\
\hline Découverte alternative & Faible & S 8 & Faible & Réalisée \\
\hline Découverte par composition & Faible & S9 & Faible & Réalisée \\
\hline Découverte par raffinement & Faible & S10 & Faible & Réalisée \\
\hline Complétude & Faible & S11 & Faible & Réalisée \\
\hline
\end{tabular}


Processus téléologique et variabilité : Utilisation de la sensibilité au contexte $\mathbf{2 2}$

\begin{tabular}{|l|l||l|l|l|}
\hline & & S12 & Faible & Réalisée \\
\hline
\end{tabular}

Table 11. Valeurs des indicateurs de la carte d'exemple.

La deuxième partie de l'évaluation du contexte (flèche 3 de la figure 3 ) correspond à l'évaluation de la situation du projet en cours. L'ingénieur de développement va ensuite utiliser ce contexte pour la prise de décision. Lorsque l'utilisateur navigue dans la carte, ses choix se situent au niveau de l'enchaînement des différents services exécutables (choix d'une section).

Pour l'illustration de notre propos, imaginons un utilisateur ayant un niveau d'expertise faible et venant de réaliser complètement l'intention 'Identifier un objectif'. Il doit maintenant choisir sa prochaine étape parmi cinq possibilités (sections S2, S3, S4, S5 et S6) comme l'indique la figure 9.

\begin{tabular}{|c|c|c|c|}
\hline \multirow{12}{*}{$\begin{array}{l}\text { Stratégie dirigée par } \\
\text { les formulaires }\end{array}$} & \multicolumn{2}{|c|}{$\begin{array}{l}\text { Stratégie dirigée par } \\
\text { les formulaires }\end{array}$} & \multirow[b]{2}{*}{ Etat de l'intention } \\
\hline & Section & Degré d'expertise & \\
\hline & S2 & Elevé & Faible realisation \\
\hline & S3 & Moyen & Faible realisation \\
\hline & S4 & Moyen & Faible realisation \\
\hline & S5 & Elevé & Réalisée \\
\hline & S6 & Faible & Réalisée \\
\hline & \multicolumn{2}{|c|}{ Stratégie } & Degré d'expertise \\
\hline & \multicolumn{2}{|c|}{ Dirigée par les formulaires } & Moyen \\
\hline & \multicolumn{2}{|c|}{ Dirigée par la structure du but } & Elevé \\
\hline & \multicolumn{2}{|c|}{ Linguistique } & Moyen \\
\hline & \multicolumn{2}{|c|}{ Ecriture libre } & Faible \\
\hline
\end{tabular}

Figure 9. Sections réalisables de l'exemple d'application.

L'utilisation de l'indicateur 'Etat de l'intention' lui permet de sélectionner soit l'exécution d'une section permettant de réaliser plus complètement la même intention (S2, S3, S4) soit celle d'une section allant à l'intention 'Ecrire un scénario' (S5, S6). Comme il a déjà réalisé complètement son intention 'Identifier un but', il choisit la deuxième possibilité et restreint donc son choix à deux sections (S5, S6). L'utilisation de l'indicateur 'Degré d'expertise' lui permet d'éliminer S5 puisqu'il lui faudrait un plus haut niveau d'expertise. Les indicateurs lui ont donc permis de sélectionner S6 pour continuer sa navigation.

Des techniques plus pointues peuvent être appliquées pour faire la sélection d'une section dans une carte en se basant sur les indicateurs, comme l'utilisation des 
23 Revue Ingénierie des Systèmes d'information.

algorithmes de graphes (Deneckere et al., 2009) ou l'application de méthodes multicritères (Deneckere et al., 2010).

\section{Discussion : Vers une notion de ligne de processus}

La variabilité croissante en génie logiciel a conduit à la notion de ligne de produits. L'ingénierie des lignes de produits correspond au développement d'applications logicielles utilisant des plateformes et de la personnalisation de masse, ce qui signifie que les points communs et les différences des applications de la ligne de produit doivent être modélisés de manière générique (Pohl et al., 2005). L'utilisation des modèles de features permet une représentation compacte de tous les produits d'une famille. Une feature est la représentation d'une caractéristique distincte d'un produit. Selon l'étape de développement à laquelle elle est utilisée, elle peut se référer à diverses données comme un besoin, un composant d'architecture ou un morceau de code d'une famille de produit (Benavides et al., 2005). Un produit d'une famille est donc défini en sélectionnant ou désélectionnant des caractéristiques selon les préférences de l'utilisateur. Les modèles de features définissent ces caractéristiques et leurs dépendances grâce à un diagramme de features associé à certaines contraintes (représentées dans un arbre ou une table). Une configuration de features est donc un ensemble de caractéristiques autorisées par le modèle de feature et qui décrit un membre de la famille de produit, autrement dit un produit spécifique (Raatikainen et al., 2005).

De la même manière qu'un Produit peut être perçu comme une ligne de Produit, c'est-à-dire une personnalisation spécifique (ou configuration) d'une famille de produit, un Processus peut également être considéré comme une ligne de Processus, donc une personnalisation spécifique (ou configuration) d'une famille de Processus (Rolland et al., 2007c).

Dans notre exemple, une carte spécifique (comme celle de la figure 6) représente alors la famille de processus et les indicateurs sont utilisés pour l'adapter au projet en cours, permettant d'obtenir par là même une ligne de processus particulière au projet.

Cette capacité de dériver une configuration de processus à partir des caractéristiques communes d'une manière reproductible est basée sur le haut niveau de variabilité des modèles intentionnels et cette configuration pendant l'exécution augmente la sensibilité au contexte des processus.

\section{Conclusion}

Nous avons montré dans ce travail que la variabilité des processus téléologiques peut être augmentée par la sensibilisation au contexte. Nous avons défini le contexte comme une composition d'indicateurs représentant les spécificités du projet. Ces 
Processus téléologique et variabilité : Utilisation de la sensibilité au contexte $\mathbf{2 4}$

indicateurs peuvent être à la fois génériques (applicables à tout processus téléologique) ou spécifiques (concernant des concepts particuliers au type de processus utilisé). Nous avons également proposé un processus d'utilisation de ces indicateurs à deux niveaux (modèle et instance). Nous avons illustré notre propos par l'intégration des indicateurs dans le méta-modèle de processus intentionnel MAP.

Nos travaux futurs concernent la définition d'une typologie plus complète pour obtenir une caractérisation du contexte plus performante, notamment en utilisant les indicateurs déduits des besoins non fonctionnels. Les stratégies d'utilisation de ces indicateurs doivent également être définies et décrites plus en détails. De plus, nous souhaitons élaborer une représentation plus agréable des indicateurs, par des moyens graphiques permettant de mieux visualiser les critères entrant en compte dans les différents points de variation des processus.

\section{Bibliographie}

(Abrahamsson, 2002) Abrahamsson, P., Salo, O., Ronkainen, J., and Warsta, J., Agile software development methods : Review and analysis. VTT Publications, 2002, Espoo.

(Amenise et al., 1993) Armenise, P., Bandinelli, S., Ghezzi, C., Morzenti, A., "A survey and assessment of software process representation formalisms", Int. Journal of Software Engineering and Knowledge Engineering, Vol. 3, No. 3, 1993.

(America et al., 2003) America P., Rommes E., Obbink J. H., "Multi-view variation modeling for scenario analysis". In $P F E^{\prime} 03,2003$, p. 44-65

(Amyot et al., 2002) Amyot D., Mussbacher G., URN : Towards a New Standard for the Visual Description of Requirements, In proceeding of 3rd Int. WS on Telecommunications and beyond : the broader applicability of SDL and MSC (2002)

(Bachmann et al., 2001) Bachmann F., Bass L., "Managing variability in software architectures". In the 2001 Symposium on Software Reusability (SSR '01), 2001, ACM Press, p. 126-132.

(Beck, 2005) Beck, K., Extreme programming eXplained : embrace change, 2nd ed. The XP Series. 2005, Boston, MA, USA, Addison Wesley.

(Benavides et al, 2005) Benavides, D., Trinidad, P., Ruiz-Cortes, A., "Automated Reasoning on Feature Models", CAISE'05, Springer-Verlag, 2005

(Bessai et al., 2008) K. Bessai, B. Claudepierre, O. Saidani and S. Nurcan, "Context-aware business process evaluation and redesign”, Proceedings of BPMDS'08, 2008.

(Boehm, 1988) Boehm, B., “A Spiral model of software development and enhancement". IEEE Computer, 1988, 21, 5, p. 61-72.

(Bosh et al., 2001) Bosch J. et al., "Variability issues in Software Product lines", $4^{\text {th }}$ international workshop on Product Family engineering (PEE-4), 2001, Bilbao, Spain

(Bradley et al., 2005) Bradley N. A., Dunlop M. D., "Toward a multidisciplinary model of context to support context-aware computing", Human-Computer interaction, 2005, Lawrence Erlbaum Associates.

(Bunt, 1997) Bunt H., "Context and dialogue control”, Proceedings of CONTEXT'97, 1997.

(Cayla, 2008) Cayla D., "Organizational Learning : A Process Between Equilibrium and Evolution”, Journal of Economic Issues, 2008, 42, 2, p.553-560. 
25 Revue Ingénierie des Systèmes d'information.

(Coutaz et al., 2002) Coutaz J., Rey G., "Recovering foundations for a theory of contextors", $4^{\text {th }}$ ICCADUI, 2002, Valenciennes, France.

(Deneckere et al., 2009) Deneckere R., Kornyshova E., Rolland C., "Enhancing the Guidance of the Intentional Model MAP : Graph Theory Application”, RCIS'09, 2009, Fes, Morocco.

(Deneckere et al., 2010) Deneckere R., Kornyshova E., "Process Line Configuration : an Indicatorbased Guidance of the Intentional Model MAP", EMMSAD'10, 2010, Tunisie.

(Dey et al., 2001) Dey, A., Abowd, G.,, Salber, D., "A conceptual framework and toolkit for supporting the rapid prototyping of context-aware applications". Human-computer Interaction, 16 2-4 (special issue on context-aware computing), 2001, p 97-166.

(Dietz, 2004) Dietz J.L.G., "Basic Notions Regarding Business Processes and Supporting Information Systems", Proceedings of BPMDS'04, 2004, Latvia, Riga, p.160-168.

(Dowson et al., 1993) Dowson, M., "Software Process Themes and Issues", IEEE Conf. on the Software Process , 1993.

(Dowson, 1988) Dowson, M., "Iteration in the Software Process", Proc 9th Int. Conf. on Software Engineering, 1988.

(Drury et al., 2008) Drury J. L., Scott S. D., "Awareness in unmanned aerial vehicle operations", International C2 journal, Geoffrey N. Hone, 2:1, 2008.

(Grosz et al., 1997) Grosz, G., Rolland, C., Schwer, S., Souveyet, C., Plihon, V., Si-Said, S., Ben Achour, C., Gnaho, C., "Modelling and Engineering the Requirements Engineering Process : An Overview of the Nature Approach", Requirements Engineering Journal, 2, 1997, p. 115131.

(Halmans et al., 2003) Halmans G., Pohl K., "Communicating the variability of a software-product family to customers", Software and System Modeling, 2(1), 2003, p.15-36

(Halmans, 2003) Halmans J., "Communicating the variability of a software product family to customers", Software and system modeling, 2003, Springer-Verlag.

(Harmsen, 1997) Harmsen F. "Situational method engineering”, Moret Ernst \& Young, 1997.

(Jarke et al., 1994) Jarke, M., Pohl, K., Rolland, C., Schmitt, J. R., "Experienced-Based Method Evaluation and Improvement : A Process Modeling Approach", CRIS series : Method and associated Tools for the Information Systems Life Cycle, 1994, North Holland.

(Keeney, 1993) Keeney, R.L. and Raiffa, H. "Decisions with Multiple Objectives : Preferences and Value Trade-Offs", Cambridge University Press, 1993

(Keeney, 1999) Keeney, R.L. "Foundations for Making Smart Decisions”, IIE Solutions, 31, No. 5,1999

(Kirsh Pinheiro et al., 2008) Kirsch Pinheiro M., Vanrompay Y., Berbers Y., "Context-aware service selection using graph matching”, Proceedings of ECOWS 2008, vol. 411, 2008.

(Kornyshova et al., 2007) Kornyshova E., Deneckère R., Salinesi C., « Method chunks selection by multicriteria techniques : an extension of the assembly-based approach", Proc. of the International IFIP WG8.1 Conference ME 07, 2007, Springer, Geneva, Switzerland.

(Kuloor et al., 2003) Kuloor C., Eberlein A., "Aspect-oriented requirements engineering for software product lines”, In ECB'03, 2003, p. 98-107.

(Liaskos et al., 2007) Liaskos S., Jiang L., Lapouchnian A., Wang Y., Yu Y., do Prado Leite J., Mylopoulos J., "Exploring the Dimensions of Variability : a Requirements Engineering Perspective". In VAMOS'07, 2007, Limerick, Ireland.

(Loucopoulos et al., 1995) Loucopoulos, P., Kavakli, E., "Enterprise Modelling and the Teleological Approach to Requirements Engineering", International Journal of Intelligent and Cooperative Information Systems, 1995.

(Malcom, 1967) Malcolm, N, “Explaining Behavior”, The Philosophical Review, 76, 1,1967.

(Mirbel et al., 2006) Mirbel I., Ralyté J., "Situational method engineering : combining assemblybased and roadmap-driven approaches", Requirements Engineering, 11(1), 2006, pp. 58-78. 
Processus téléologique et variabilité : Utilisation de la sensibilité au contexte $\mathbf{2 6}$

(Ngo-The et al., 2005) Ngo-The, A., Ruhe, G., "Decision Support in Requirements Engineering", In Engineering and Managing Software Requirements, Ed. By A. Aurum and C. Wohlin, 2005.

(Nurcan, 2004) Nurcan S., "Business Process Modeling for developing Process Oriented IT Systems", IRMA'04, 2004, New Orleans, USA.

(Pohl et al., 2005) Pohl K., Böckle G., van der Linden F., "Software product line engineering : foundations, principles and techniques", 2005, Springer, Berlin Heidelberg New York.

(Prakash et al., 2006) Prakash N., Rolland C., "Systems Design for requirements expressed as a map", Proc. of the conference IRMA 06, 2006, Washington DC.

(Raatikainen et al., 2005) Raatikainen, M., Soininen, T., Männistö, T. and Mattila, A., "Characterizing configurable software product families and their derivation", Software Process : Improvement and Practice, Special Issue on Software Variability : Process and Management, Volume 10, Issue 1, January/March 2005, pages 41-60

(Ralph, 2008) Ralph, P., Wand, Y., "A Teleological Process Theory of Software Development", JAIS Theory Development Workshop. 8, 23, 2008.

(Ralyté et al., 1999) Ralyté, J., Rolland, C., Plihon, V., "Method Enhancement with Scenario Based Techniques". CAISE'99, Springer-Verlag, Heidelberg, Germany, 1999, p. 103-118.

(Ralyté, 2001) Ralyté, J., Rolland, C. "An Assembly Process Model for Method Engineering", CAISE'01, Springer-Verlag, 2001

(Rey et al., 2002) Rey, G., Coutaz, J., "Le Contexteur : une abstraction logicielle pour la réalisation de systèmes interactifs sensibles au contexte ». IHM'02, 2002, p. 105-112.

(Rolland, 1998) Rolland, C., “A Comprehensive View of Process Engineering”, CAISE'98, Springer-Verlag, 1998.

(Rolland, 2007) Rolland C., "Capturing system intentionality with maps", in the book Conceptual Modelling in Information Systems Engineering, 2007.

(Rolland et al., 1999) Rolland C., Prakash N., Benjamen A., "A Multi-Model View of Process Modelling”. Requirements Engineering. Vol. 4, N 4. 1999, Springer-Verlag London Ltd.

(Rolland et al., 2000) Rolland, C., Nurcan, S., Grosz G., "A Decision Making Pattern for Guiding the Enterprise Knowledge Development Process", Journal of Information and Software Technology, Elsevier, 42, 2000, p. 313 - 331

(Rolland et al., 2007b) Rolland C. , Prakash N., "On the Adequate Modeling of Business Process Families", BPMD'07, 2007, Trondheim, Norway.

(Rolland et al., 2007c) Rolland C., Prakash N., Kaabi R. S., "Variability in Business Process Families", Information Resources Management Association (IRMA), 2007.

(Rosemann et al., 2006) Rosemann M., Recker J., "Context-aware process design : exploring the extrinsic drivers for process flexibility”, CAISE'06, workshops and doctoral consortium, 2006, Luxembourg, Namur University Press., p 149-158.

(Rosen et al., 2008) Rosen M. A., Fiore S. M., Salas E., Letsky M., Warner N., "Tightly coupling cognition : understanding how communication and awareness drive coordination in teams", International C2 journal, 2:1, 2008.

(Roy, 1996) Roy, B. "Multicriteria Methodology for Decision Aiding", Dordrecht, Kluwer Academic Publishers, 1996

(Roy, 2005) Roy, B., "Paradigms and challenges, Book chapter, In Multiple Criteria Decision Analysis - State of the Art Survey", 2005, Springer. editor(s) J. Figueira, S. Greco, M. Ehrgott.

(Royce, 1970) Royce, W. W., "Managing the development of large software systems : concepts and techniques", In Proceedings of Wescon, 1970.

(Ruhe, 2003) Ruhe, G., "Software Engineering Decision Support - Methodology and Applications". In : Innovations in Decision Support Systems, Ed. by Tonfoni and Jain, International Series on Advanced Intelligence, Vol. 3, 2003, p. 143-174. 
27 Revue Ingénierie des Systèmes d'information.

(Salber et al., 1998) Salber, D., Dey, K. A., Abowd, D. G., "Ubiquitous Computing : Defining an HCI research agenda for an emerging interaction paradigm". In Georgia Tech GVU technical report, 1998.

(Salifu, 2007) Salifu, M., Yu, Y., Nuseibeh, B., "Specifying monitoring and switching problems in context". Proc. 15th Int. Conference on Requirements Engineering RE'07, 2007

(Schilit et al., 1994) Schilit B., Adams N., Want R.. "Context-aware computing applications", IEEE WMCSA'94, Santa Cruz, CA, US, 1994, p. 89-101.

(Svanberg, 2001) Svanberg, "On the notion of variability in software product lines", working IEEE/IFIP conference on software architecture, 2001.

(Taylor, 1964) Taylor, Ch., "The Explanation of Behaviour”, 1964, London, Routledge.

(Van de Ven, 1995) Van de Ven A.H., Poole, M.S., "Explaining Development and Change in Organizations", The Academy of Management Review, Vol. 20, No. 3, 1995, p. 510-540.

(Van Gurp, 2000) Van Gurp J., "Variability in Software Systems, the key to Software Reuse", Licentiate Thesis, 2000, University of Groningen, Sweden.

(Van Lamsweerde, 2009). van Lamsweerde A., "Reasoning About Alternative Requirements Options", Conceptual Modeling : Foundations and Applications, 2009, p. 380-397

(Van Slooten et al., 1996) Van Slooten K., Hodes B., "Characterising IS development projects", IFIP WG8.1 Conference on Method Engineering, 1996.

(Veblen, 1898) Veblen, Th., "Why is Economics not an Evolutionary Science?" The Quarterly Journal of Economics, 12, 4,1898, p. 373-397.

\section{Annexe : Plus d'informations sur les auteurs}

Pour plus d'informations sur les auteurs, merci de scanner les codes suivants.

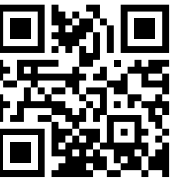

Rébecca Deneckère

Elena Kornyshova

Pour scanner, vous pouvez télécharger le logiciel suivant sur votre mobile : http://www.lynkware.com/support_devices.php

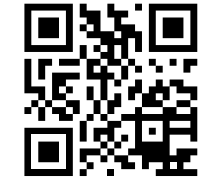

